trum of $T$ are mutually exclusive, and consequently we have a contradiction. Hence the theorem is proved. The following corollary is an immediate consequence of the theorem:

The numerical range of a unitary operator in a Hilbert space is closed if and only if the spectrum of the unitary operator consists entirely of the point spectrum.

\title{
REFERENCES
}

1. B. Sz.Nagy, Spektraldarstellung linearer Transformationen des Hilbertschen Raumes, Ergebnisse der Mathematik vol. 5, Berlin, 1942.

2. M. H. Stone, Linear transformations in Hilbert space and their applications to analysis, Amer. Math. Soc. Colloquium Publications, vol. 15, New York, 1932.

Sacramento State College

\section{ON POSTULATES FOR GENERAL QUANTUM MECHANICS}

\section{B. LOWDENSLAGER ${ }^{1}$}

A collection of postulates for the observable quantities in a general quantum mechanical system has been given by Segal, [4]. In this system, the observables form a real Banach space $S$ which is partially ordered by a closed convex positive cone $P$, and which has a unit element $e$ interior to the positive cone, all satisfying $P \cap(e-P)$ $=S_{1}(e) \cap S_{1}(0)$, where $S_{r}(x)$ is the solid sphere of radius $r$, center $x$. Alternatively, $S$ is a partially ordered linear space with an element $e>0$ which is a Banach space under the norm $\|x\|=\min \{\lambda:-\lambda e$ $\leqq x \leqq \lambda e\}$, where the minimum is attained. Let us call such a space a partially ordered Banach space with unit. We shall show that any such Banach space $S$ with order unit $e$ can be given the algebraic structure postulated by Segal, in possibly different ways. In this proof it is very useful to have an alternative formulation of Segal's postulates stated in terms of the partial ordering alone. We are then able to give examples of such systems with various pathologies and to classify the three dimensional ones. Finally we shall give a necessary and sufficient condition that a system of operators satisfying these postulates be a $C^{*}$ special Jordan algebra: the system of hermitean elements of a $C^{*}$ algebra.

Definition. A spectral function $L$ on a partially ordered Banach space $S$ with order unit $e$ is a function assigning to each point $x \in S$ a

Received by the editors February 27, 1956.

1 This work was done under contract Nonr-222(37) with the O.N.R. 
closed linear subspace $L(x)$ of $S$ such that: $1 . e \in L(x), 2 . L(x)$ is a lattice in the ordering it inherits as a subspace of the partially ordered Banach space $S, 3 . v \in L(x)$ if and only if $L(v)$ is a sublattice of $L(x)$, and 4. the function sending $x \in S$ into $x \bigvee 0 \in L(x)$ is continuous in the norm on $S$.

THEOREM 1. Let $S$ be a partially ordered Banach space with order unit $e$, and let $L(x)$ be a spectral function on $S$. There exists a unique polynomial operation $x \rightarrow p(x)$ defined for $x \in S, p$ a real polynomial, satisfying Segal's postulates such that in Segal's terminology $P$ is the positive cone, $e$ is the unit and each $L(x)$ is a commutative subsystem.

Proof. By Theorem 4.1 of [1], for each $L(x)$ there exists a unique compact Hausdorff space $X$ such that $L(x)$ is order and linear isomorphic to the set of all real continuous functions on $X, C(X)$, and $e$ corresponds to the function identically 1 . Define, for any real polynomial $p, p(x)$ to be the element in $L(x)$ which is represented by the function $\{p(x(t)) \mid t \in X\}$, where $\{x(t) \mid t \in X\}$ represents $x$. Since $p(x) \in L(x), L(p(x))$ is a sublattice of $L(x)$ and, $q(p(x))=(q p)(x)$, where $q$ is any other such polynomial. In fact, the representation space for $L(p(x))$ must be an identification space of $X$ in an obvious way. We can now verify Segal's postulates. Postulate $I$ is that $S$ is a real linear space, with, for every element $x$ an operation forming polynomials in $x$ satisfying $(q p)(x)=q(p(x))$. Postulate II requires that $S$ be a Banach space satisfying $\left\|u^{2}-v^{2}\right\| \leqq \operatorname{Max}\left\{\left\|u^{2}\right\|,\left\|v^{2}\right\|\right\}$, $\left\|u^{2}\right\|=\|u\|^{2},\left\|\sum u^{2}\right\| \leqq\left\|\sum^{\prime} u^{2}\right\|$, if $\sum$ is a summation over a finite subset of the finite summation $\Sigma^{\prime}$, and $u^{2}$ continuous in $u$. The first four requirements of postulate II follow immediately from the definition of the norm in terms of the order, when it is remarked that an element is positive if and only if it is a square. The continuity of $u^{2}$ in $u$ follows from the continuity of $u \vee 0$ and $u \wedge 0$ in $u$. The fact that the multiplication is unique follows from Corollary 5 of [2], applied to the commutative formally real Banach algebra $L(x)$.

REMARK. The converse of Theorem 1 is true also: Then $L(x)$ should be chosen to be the commutative subsystem generated by $x$ and $e$. Corollary 1.1 of [4] shows that this $L(x)$ is isomorphic algebraically and metrically to the system of all continuous functions on a compact Hausdorff space. The positive elements of $S$ are the sums of squares, and the main result of [5] is that the natural order in $L(x)$ as a function algebra is the inherited order of $S$.

COROLlaRY. In any partially ordered Banach space with order unit $e$ there is at least one spectral function $L$ satisfying our theorem, and hence at least one polynomial operation satisfying Segal's postulates. 
Proof. For $L(x)$ choose the closed linear subspace spanned by $x$ and $e$. Unless $x=\lambda e, L(x)$ is a 2-dimensional space with a partial ordering generated by a closed cone with interior. Such a space must be a vector lattice of the type desired. In fact, the continuity of $x \vee 0$ in $x$ follows from the simple geometric interpretation of $\|x\|$ and $x \vee 0$ in the two dimensional plane spanned by $x$ and $e$. If $u \in L(x)$, $u$ is a linear combination of $x$ and $e$, and $L(u)$ is clearly a sublattice of $L(x)$.

EXAmple 1. A simple complete enumeration of all possible 3dimensional systems is possible. A three dimensional vector lattice satisfying our postulates must have a trihedral cone as positive cone. Any interior point will serve as unit. Thus, unless the cone is trihedral, $L(u)$ must be two dimensional, and hence of the type described in the corollary. If the product $a \circ b=1 / 2\left\{(a+b)^{2}-a^{2}-b^{2}\right\}$ is bilinear in $a$ and $b$, the system is a formally real Jordan algebra, described in [3]. Perusal of the classification achieved there shows that the only new possibility for $P$ is an elliptic cone, with $e$ any point on the axis. Such a system is isomorphic to the special Jordan algebra of all $2 \times 2$ real symmetric matrices with our multiplication. Thus, for every other closed proper convex cone and choice of $e$ in the interior, we get a system in which the product above is not distributive. ${ }^{2}$

EXAmple 2. Let $H$ be a real Hilbert space, and $S$ the space whose points are pairs $(h, t)$ where $h \in H, t$ is real, with the obvious scalar multiplication and addition. Let $P$ consist of the pairs $(h, t)$ with $\|h\| \leqq t$, let $e=(0,1)$ and $\|x\|=\inf \{\lambda:-\lambda e \leqq x \leqq \lambda e\}$. First we remark that $S$ is not a lattice in this ordering unless the dimension of $H$ is 1 . In fact, consider $X_{+}=(h, 0), X_{-}=(-h, 0)$ where $\|h\|=1$. If $X_{+} \wedge X_{-}$ exists, it must be of the form $(0, t)$ since $P$ is symmetric with respect to reflection about the $t=0$ subspace; and clearly $t=-1$. If $g \perp h$, $\|g\|=1$ for some $g \in H$, then $Z=\left(g / 2,-(3 / 2)^{1 / 2}\right)$ satisfies $Z \leqq X_{+}$, $Z \leqq X_{-}$but not $Z \leqq(0,-1)$. A closed linear subspace of $S$ containing $e$ must be of the form $S^{\prime}$ where $S^{\prime}$ is constructed from a closed linear subspace $H^{\prime}$ of $H$ as $S$ was constructed from $H$. Thus the only commutative subalgebras in any system of multiplication defined on $S$ must be 2-dimensional, whatever the dimension of $S$. Thus our system gives the only possible definition of a squaring operation. $S$ is

2 S. Sherman had announced the existence of such an example before this paper was written, although these results were obtained independently of his. When Professor Sherman kindly lent me a manuscript of his paper, I found that he had by an explicit calculation a special case of Example 1.

This work is the subject of the recent paper, On Segal's postulates for general quantum mechanics, Ann. of Math. vol. 64 (1956) pp. 593-601 by S. Sherman. 
interesting also because it is reflexive as a Banach space, while an infinite dimensional $C^{*}$ algebra never is.

There exist many linear isometric order preserving representations of a partially ordered Banach space $A$ with order unit $e$ onto a set of hermitean operators on a Hilbert space such that $e$ goes into the identity. It is nevertheless simple to give a condition on the canonical operation $x \rightarrow x \vee 0$ which will insure that a system of hermitean operators along with the associated product $a \circ b$ be the collection of self-adjoint operators on a $C^{*}$ algebra, with $a \circ b=1 / 2(a b+b a)$.

Theorem 2. Suppose a partially ordered vector space $S$, with order unit e, and spectral function $L$ is a collection of hermitean operators on a Hilbert space, $e$ is the identity operator, and the range of the operator $x \vee 0$ is always orthogonal to that of $x \wedge 0$. Then the product $a \circ b$ is the Jordan product $1 / 2(a b+b a)$, and $S+i S$ is a $C^{*}$ algebra.

Proof. Let $L(x)$ be linearly and order isomorphic to the set of continuous real valued functions on the compact Hausdorff space $X$. Then since $x=x \vee 0+x \wedge 0$, and, by hypothesis, the projection on the range of $x \vee 0$ reduces $x$, we must have $x \vee 0-x \wedge 0=\left(x^{2}\right)^{1 / 2}$, so that Theorem 6 of [2] applies, and the isomorphism between $C(x)$ and $L(x)$ preserves products. This implies that the set $S+i S$ is closed under squaring, and hence, by a polarization identity, is closed under the ordinary product $a b$, and is a $C^{*}$ algebra.

\section{REFERENCES}

1. R. V. Kadison, $A$ representation theory for commutative topological algebra, Memoirs of the American Mathematical Society, no. 7, 1951.

2. - A generalized Schwarz inequality and algebraic invariants for operator algebras, Ann. of Math. vol. 56 (1952) pp. 494-503.

3. P. Jordan, J. v. Neumann, and E. Wigner, On an algebraic generalization of the quantum mechanical formalism, Ann. of Math. vol. 35 (1934) pp. 29-64.

4. I. E. Segal, Postulates for general quantum mechanics, Ann. of Math. vol. 48, (1947) pp. 939-948.

5. S. Sherman, Non-negative observables are squares. Proc. Amer. Math. Soc. vol. 2 (1951) pp. 31-33.

University of CALifornia, Berkeley 